\title{
AC and DC Bias-Temperature Stability of Coplanar Homojunction a-InGaZnO Thin-Film Transistors
}

\author{
Eric Kai-Hsiang Yu*, Katsumi Abe ${ }^{* *}$, Hideya Kumomi** and Jerzy Kanicki* \\ *Dept. of Electrical Engineering and Computer Science, University of Michigan, Ann Arbor, MI, United States \\ **Materials and Structures Lab, Tokyo Institute of Technology, Midori-ku, Yokohama, Japan
}

\begin{abstract}
We fabricated coplanar homojunction a-IGZO TFTs that are highly stable under $A C$ and DC bias-temperature-stress. For TFTs of the size $W / L=60 \mu m / 10 \mu m$, the stress-induced threshold voltage shifts are all within $-0.35 \mathrm{~V}$. A comprehensive investigation of $A C$ BTS stress polarity, pulse width, and duty cycle dependence is presented. We find that higher frequency of bipolar AC pulses increases the device instability, while lower duty cycle values have the opposite effect.
\end{abstract}

\section{Author Keywords}

Oxide; TFT; a-IGZO; instability; stress; AC; BTS

\section{Introduction}

The amorphous In-Ga-Zn-O thin-film transistor (a-IGZO TFT) is promising as the backplane technology for next-generation ultrahigh definition displays and low-power mobile displays [1][2]. Current-temperature stress (CTS) and bias-temperature stress (BTS) stability are critical factors for flat-panel display lifetime; having reliable TFTs will greatly reduce pixel circuit complexity. For constant CTS conditions mimicking AM-OLED operation, stress-induced threshold voltage shift $\left(\Delta \mathrm{V}_{\text {th }}\right)$ of a-IGZO TFT is found to be much smaller $(0.2 \mathrm{~V})$ than that of a-Si:H TFT $(>1.8$ V) [3]. In previous work from our group [4], we found that the DC BTS-induced $\Delta \mathrm{V}_{\text {th }}$ instability for the a-IGZO TFT is significantly lower than the a-Si TFT, in agreement with other works [5][6]. For the a-IGZO TFT, positive and negative DC BTS (dark or illuminated) are all well documented [7][8]. However, $\mathrm{AC} \mathrm{BTS}$ is required to accurately represent common flat-panel display addressing conditions for lifetime estimation, and the literature in this area is limited in terms of stressing conditions investigated [9-11]. Furthermore, AC BTS $\Delta \mathrm{V}_{\text {th }}$ instability data reported in the literature are all on the order of several volts.

Our objective is to fabricate highly reliable bottom-gate coplanar homojunction a-IGZO TFTs and evaluate their electrical stability under AC and DC BTS. We present a comprehensive set of data on the AC electrical stability using various test conditions: polarity, pulse width, and duty cycle. Our devices are all very stable under both AC and DC BTS and we observe no $\Delta \mathrm{V}_{\text {th }}$ greater than $-0.35 \mathrm{~V}$.

\section{Experimental}

Device Fabrication: The Mo gate (100nm) is sputtered on a glass substrate and patterned by dry etching. Using plasmaenhanced chemical vapor deposition (PECVD), a-SiOx (200nm) is deposited as gate insulator at $340^{\circ} \mathrm{C}$. The $40 \mathrm{~nm}$-thick a-IGZO active layer is DC-sputtered and defined using dilute hydrochloric acid. To protect the channel, a-SiOx stopper layer $(300 \mathrm{~nm})$ is deposited by PECVD at $285^{\circ} \mathrm{C}$ and dry etched. The channel width (W) and length (L) of the TFTs fabricated are W/L $=60 \mathrm{um} / 10 \mathrm{um}$ and this is defined by the size of the stopper layer. The a-SiONx passivation layer $(300 \mathrm{~nm})$ is deposited by PECVD at $250^{\circ} \mathrm{C}$. The source/drain (S/D) contact via are formed by dry etching. The Mo $\mathrm{S} / \mathrm{D}$ electrodes $(100 \mathrm{~nm})$ are then sputtered and wet-etched. After device processing, the TFTs undergo one final annealing step of $290^{\circ} \mathrm{C}$ for 60 minutes. The device top-view micrograph and crosssection diagram are shown in Fig. 1.

Measurement and Parameter Extraction: The a-IGZO TFT transfer characteristics $\left(\mathrm{ID}_{\mathrm{D}}-\mathrm{V}_{\mathrm{GS}}\right)$ between $\mathrm{V}_{\mathrm{GS}}=-10 \mathrm{~V}$ to $10 \mathrm{~V}$ in linear $\left(\mathrm{V}_{\mathrm{DS}}=0.1 \mathrm{~V}\right)$ and saturation $\left(\mathrm{V}_{\mathrm{DS}}=15 \mathrm{~V}\right)$ regions are measured using an Agilent B1500A semiconductor analyzer and shown in Fig. 2 for temperature $\mathrm{T}=70^{\circ} \mathrm{C}$. The TFT field-effect mobility $\left(\mu_{\mathrm{FE}}\right)$ and threshold voltage $\left(\mathrm{V}_{\mathrm{th}}\right)$ at $\mathrm{V}_{\mathrm{DS}}=0.1 \mathrm{~V}$ are extracted by linearly fitting the transfer curve to the equation:

$$
I_{D}=\frac{W}{L} C_{O X} \mu_{F E}\left(V_{G S}-V_{t h}\right) V_{D S}
$$

between the $80 \%$ and $20 \%$ maximum drain current points and extrapolating the $\mathrm{x}$-intercept of the fit. For the TFT shown in Fig. 2 , the device has the parameters $\mu_{\mathrm{FE}}=10.9 \mathrm{~cm}^{2} / \mathrm{Vs}, \mathrm{V}_{\mathrm{th}}=-1.03 \mathrm{~V}$, and sub-threshold swing (S) of $220 \mathrm{mV} / \mathrm{dec}$ An example of the AC BTS performed in this study is plotted in the same figure as a dotted line, showing the excellent reliability of the TFT over an extended period of stressing.

Bias-Temperature Stress: Device measurement and stressing are done at $70^{\circ} \mathrm{C}$ in the dark. The B1500A and Agilent $8114 \mathrm{~A}$ pulse generator are connected to an Agilent E5250A switching matrix, which is controlled by the B1500A built-in Agilent EasyExpert software. The E5250A switches its output between the $8114 \mathrm{~A}$ for $\mathrm{AC}$ stressing and the B1500A for

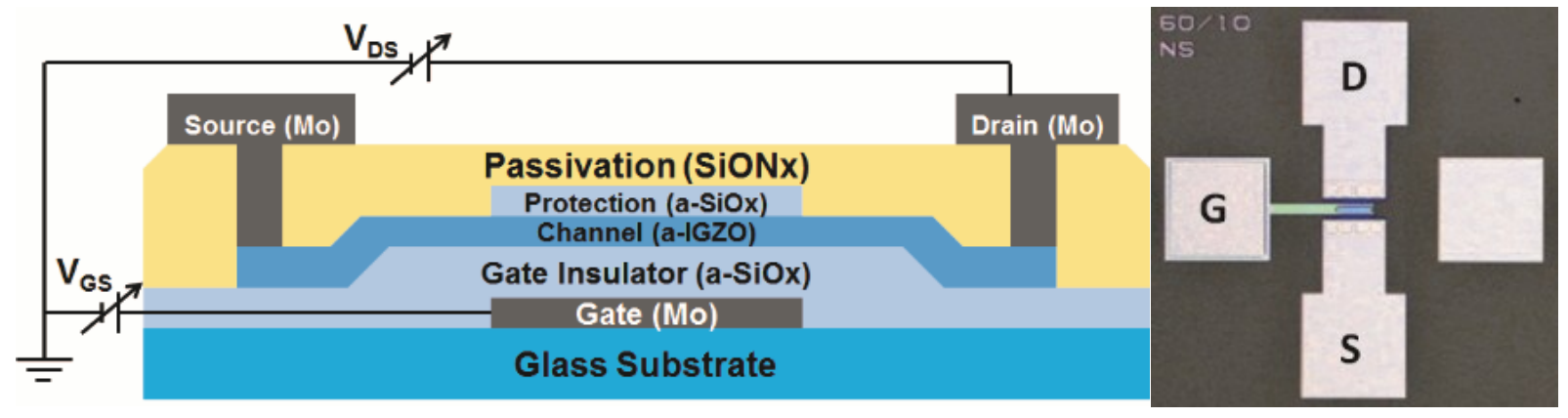

Figure 1. Cross-section and top-view of the bottom-gate coplanar homojunction a-IGZO TFT in this investigation. 


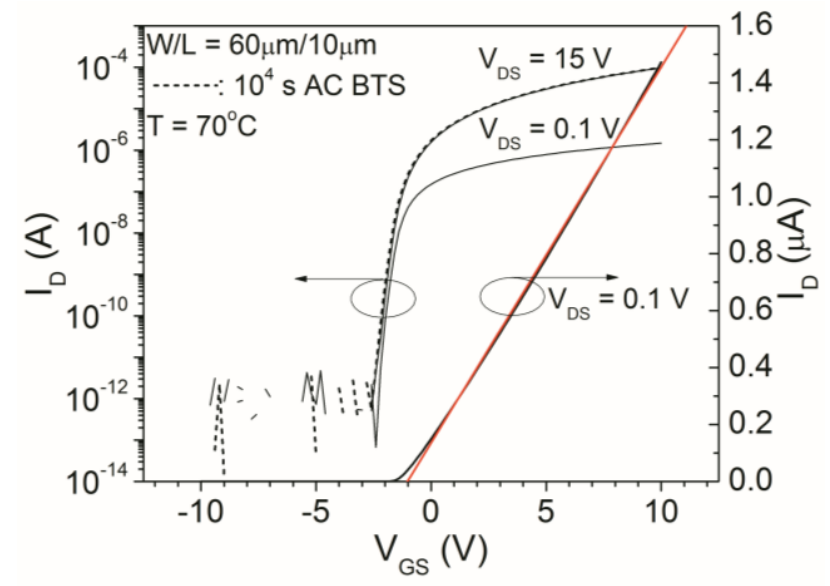

Figure 2. The transfer characteristics of the coplanar homojunction a-IGZO TFTs. Dashed lines indicate the transfer curve shift after $10^{4} \mathrm{~s}$ of AC BTS.

transfer characteristics measurements. The three types of AC BTS waveforms used in this study are shown in Fig. 3: positive unipolar $\left(\mathrm{V}_{\mathrm{G}-\text { Stress }}=0\right.$ to $\left.+20 \mathrm{~V}\right)$, negative unipolar $(0$ to $-20 \mathrm{~V})$, and bipolar $(-20 \mathrm{~V}$ to $+20 \mathrm{~V})$. During stressing, the drain and source are tied together and grounded $\left(\mathrm{V}_{\mathrm{DS}}=0 \mathrm{~V}\right)$ to ensure a uniform distribution of the electric field across the channel. The device stressing is interrupted at set time steps to measure the transfer characteristics. This repeats until overall accumulated stress time reaches $10^{4} \mathrm{~s}$. Accumulated stress time is defined as the total amount of time a non-zero stress bias (positive or negative) is applied to the gate. A different TFT on the same wafer is used for each stress condition.

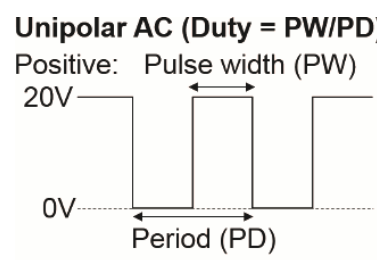

Negative:

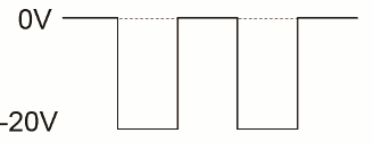

Bipolar AC (Duty $\left.=t_{H I} / P D\right)$

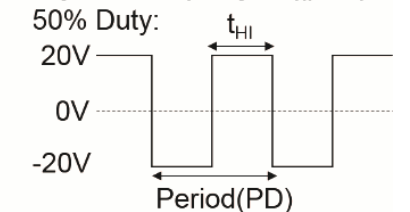

$20 \%$ Duty:

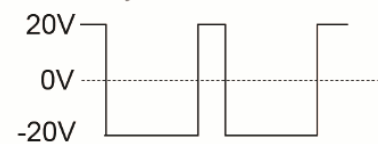

$10 \%$ Duty:

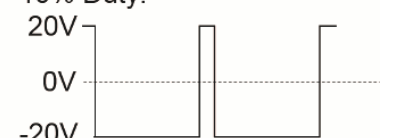

Figure 3. The unipolar and bipolar AC BTS waveforms used in this investigation.

\section{Results and Discussion}

In Fig. 4, we compare and examine the relationship between DC and $\mathrm{AC}$ bipolar (pulse width $\mathrm{PW}=10 \mathrm{~ms}$ ) BTS instability. We see that the sum of the positive and negative DC BTS $\Delta \mathrm{V}_{\text {th }}$ exceeds that of the bipolar AC BTS, which means that DC BTS cannot be used to help us understand $\mathrm{AC}$ behavior. The bipolar AC BTS $\Delta \mathrm{V}_{\text {th }}$ actually falls between the DC BTS values. For the a-Si TFT, it has been shown that bipolar AC BTS can be estimated by summing the $\Delta \mathrm{V}_{\text {th }}$ of positive and negative unipolar AC BTS segments of equal duration [12]. We confirm that the same also applies to the a-IGZO TFT in Fig. 5, where the sum of the unipolar positive and negative AC BTS tracks the bipolar AC BTS curve very well.

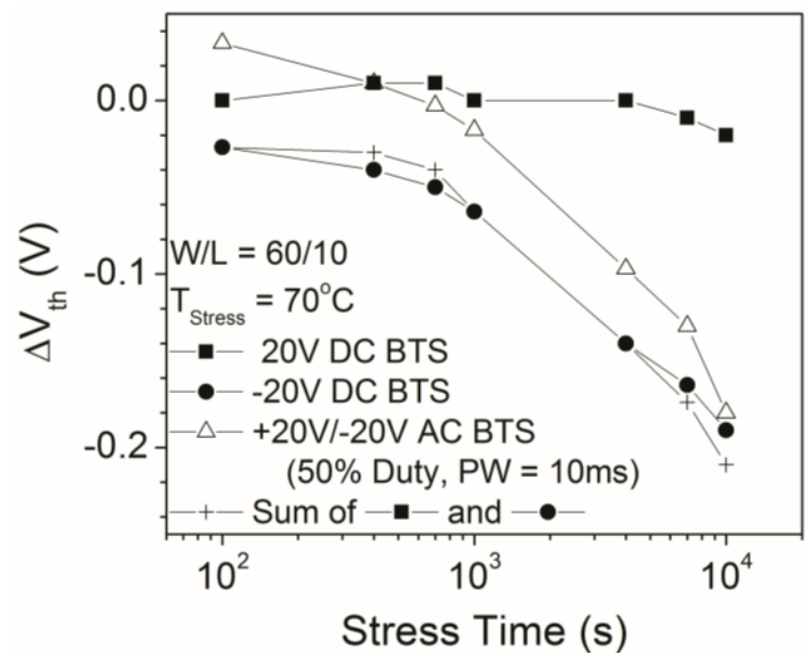

Figure 4. $\Delta V_{\text {th }}$ vs. accumulated stress time for bipolar $A C$ and DC BTS of the a-IGZO TFT (W/L $=60 / 10)$. Sum of positive and negative DC BTS is also shown.

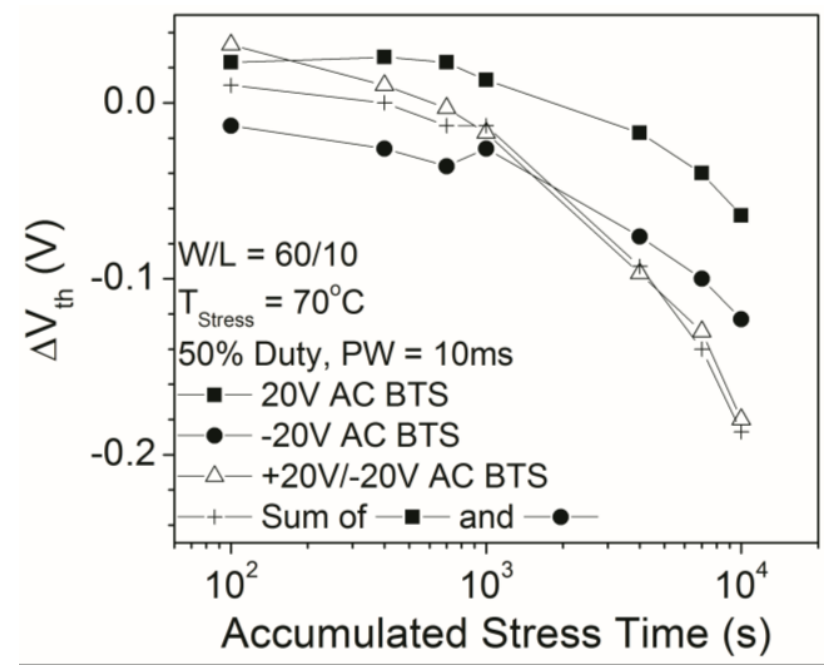

Figure 5. The bipolar AC BTS $\Delta \mathrm{V}_{\text {th }}$ can be approximated by adding the positive and negative unipolar AC BTS segments of equal accumulated stress time.

In Fig. 6, we change the pulse width for both (a) positive $(+20 \mathrm{~V})$ and (b) negative $(-20 \mathrm{~V})$ unipolar AC BTS. In the case of $+20 \mathrm{~V}$ AC BTS, the $\Delta \mathrm{V}_{\text {th }}$ is larger for shorter pulse widths. We also observe that for all pulse widths, the $\Delta \mathrm{V}_{\text {th }}$ initially enters a phase of positive shift for up to $1000 \mathrm{~s}$ of stressing, and then moves towards the negative direction for longer stressing. This phenomenon is similar to the stress-recovery transition reported for positive BTS of a-IGZO TFTs in the literature [11]. We do not see any sort of transition for $-20 \mathrm{~V}$ unipolar AC BTS, the $\Delta \mathrm{V}_{\text {th }}$ of which are entirely negative and their magnitudes increase with increasing pulse width.

The relationship between pulse width and instability for $-20 \mathrm{~V}$ AC BTS is further examined when we normalize $\Delta V_{\text {th }}$ for various pulse widths to the DC BTS shift, and this is shown in Fig. 7. 


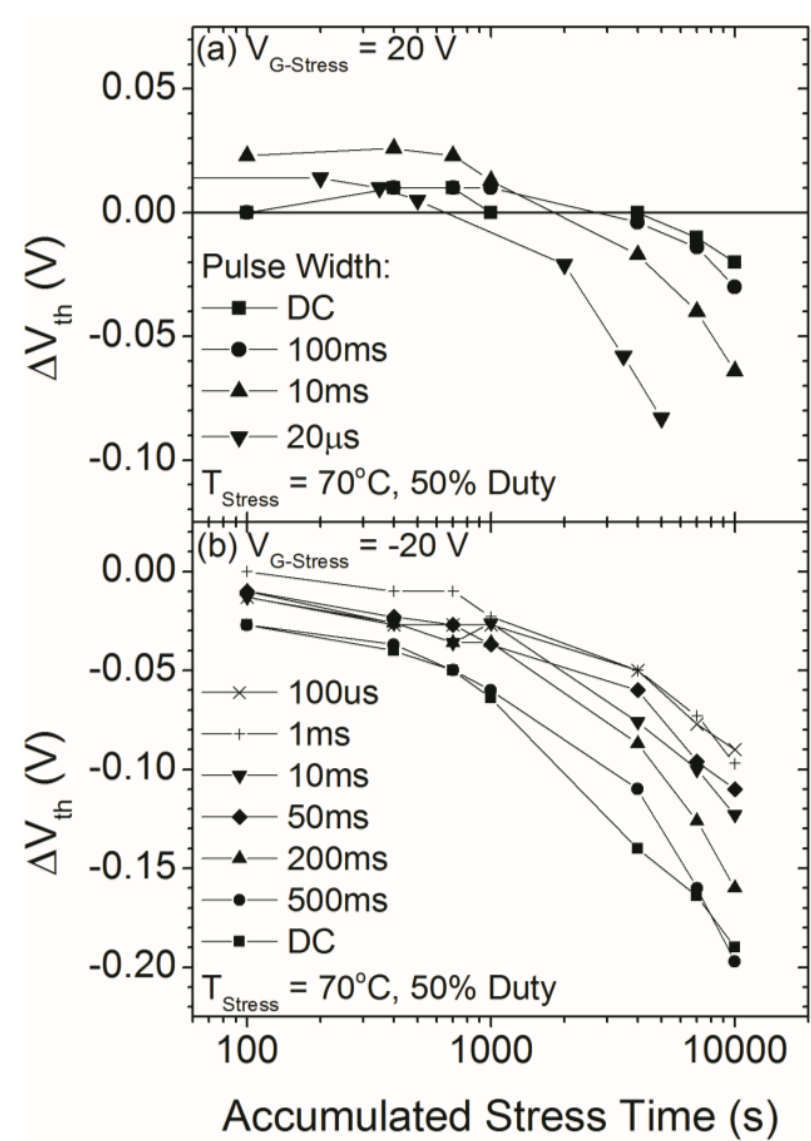

Figure 6. $\Delta \mathrm{V}_{\text {th }}$ vs. accumulated stress time for (a) positive and (b) negative unipolar AC BTS with varying pulse width.

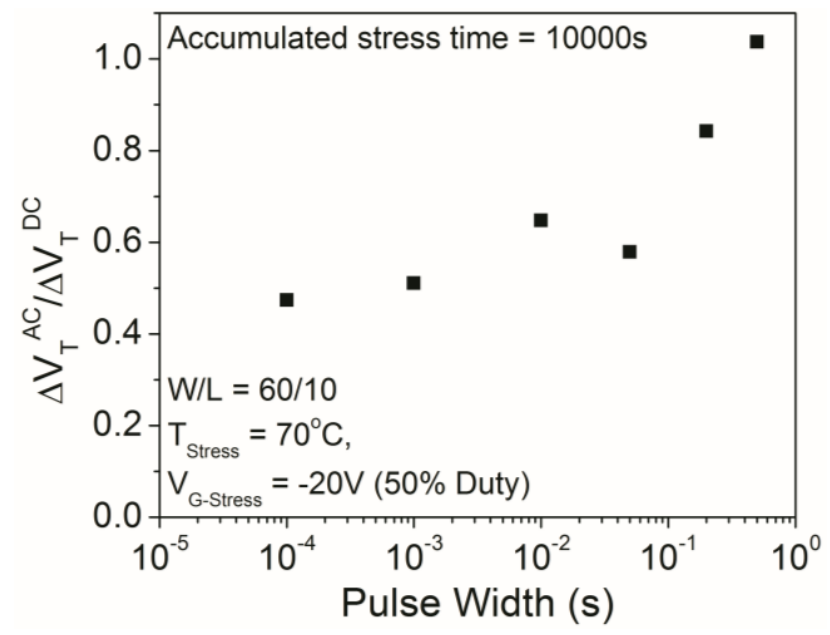

Figure 7. $\Delta \mathrm{V}_{\text {th }}$ for $-20 \mathrm{~V}$ unipolar $\mathrm{AC}$ BTS at various pulse widths, normalized to the $-20 \mathrm{~V}$ DC BTS value.

In Fig. 8, duty cycle dependence is investigated for the bipolar AC BTS with pulse period PD $=20 \mathrm{~ms}(50 \mathrm{~Hz})$, the waveforms for which are described in Fig. 3. In the case of the bipolar AC gate stress, a lower duty cycle value such as Duty $=10 \%$ means that within a fixed period, positive bias segments occupy $10 \%$ and negative bias segments occupy $90 \%$ of the time frame. We observe that the TFT is more stable when operated at lower duty cycle.

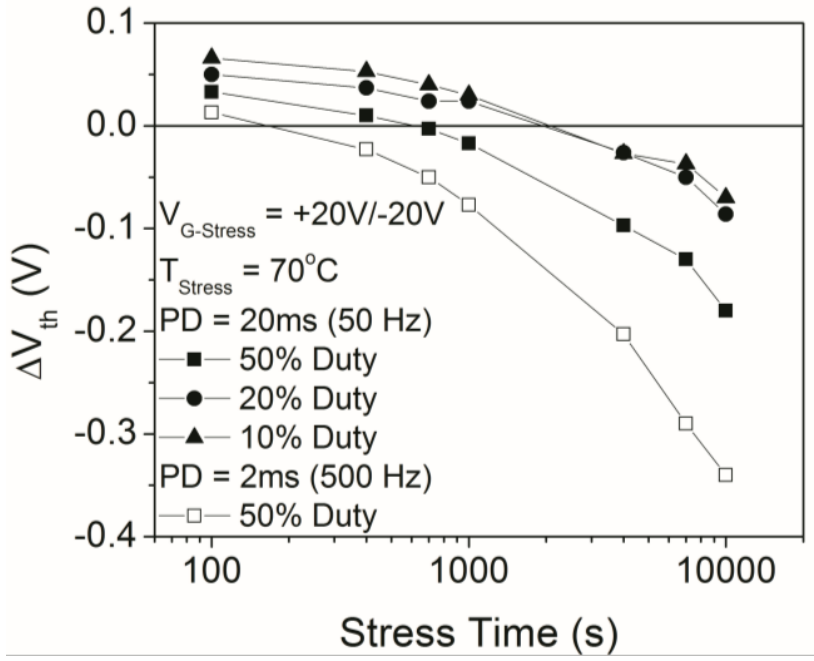

Figure 8. Bipolar AC BTS for various duty cycles at $50 \mathrm{~Hz}$ operation. The instability for $50 \%$ duty at $500 \mathrm{~Hz}$ is also included for comparison.

Lastly, to study the impact of operation frequency on the stability of a-IGZO TFT, bipolar AC gate stress is applied for frequencies ranging from $50 \mathrm{~Hz}$ to $500 \mathrm{~Hz}$ at $50 \%$ duty cycle. We see from Fig. 9 that bipolar AC instability does have frequency dependence and that higher frequencies show greater $\Delta \mathrm{V}_{\text {th }}$ instability. This is especially the case for bipolar gate pulse of $500 \mathrm{~Hz}$ (corresponding to $480 \mathrm{~Hz}$ display refresh rate), for which the $\Delta \mathrm{V}_{\text {th }}=-0.35 \mathrm{~V}$ after $10^{4} \mathrm{~s}$ is the greatest threshold voltage shift observed within this study. Clearly, AC BTS instability needs to be taken into consideration when using aIGZO TFTs for high-refresh rate displays. However, it may be possible to reduce the instability by controlling the duty cycle such that the pulse width of the positive ON-pulses are as short as the dynamic response of the a-IGZO TFT permits.

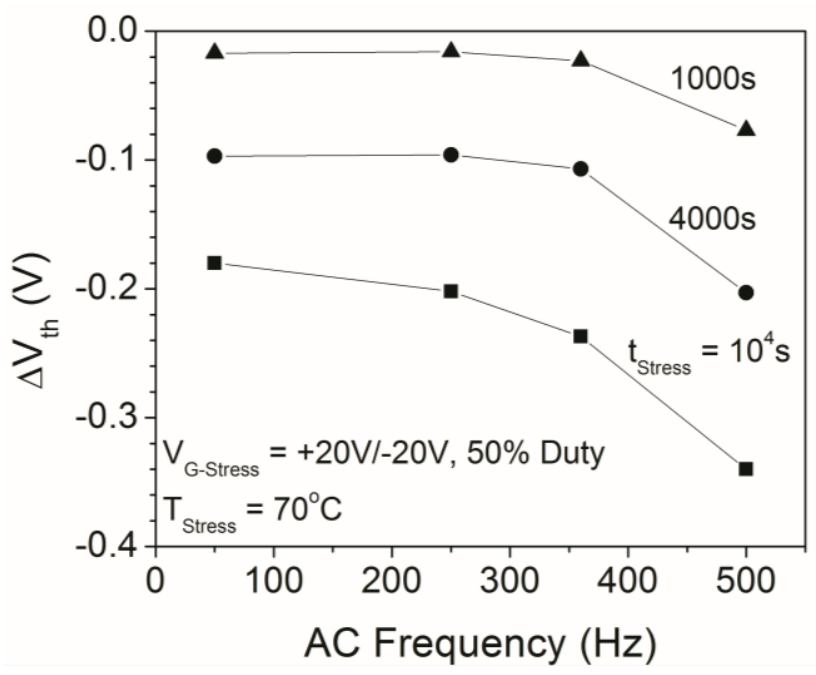

Figure 9. Operation frequency dependence of bipolar $A C$ BTS $\Delta \mathrm{V}_{\text {th }}$ for a-IGZO TFTs. Frequencies of $50 \mathrm{~Hz}, 250 \mathrm{~Hz}$, $360 \mathrm{~Hz}, 500 \mathrm{~Hz}$ are evaluated. 


\section{Conclusion}

We have fabricated high-performance and highly stable bottomgate coplanar homojunction a-IGZO TFTs that have a-SiONx as passivation layer. A comprehensive AC BTS study is conducted on the TFTs, and they have been demonstrated to be very reliable under a wide variety of stressing conditions at $70^{\circ} \mathrm{C}$.

We find that for our TFTs, the bipolar AC BTS instability timeevolution can be well-described by a simple sum of the positive and negative unipolar AC BTS instability, but not the sum of the DC BTS instabilities. The AC pulse width dependence of the threshold voltage shift is thoroughly investigated. We find that negative unipolar pulses exhibit larger (more negative) $\Delta \mathrm{V}_{\text {th }}$ shift for longer pulse widths. For positive unipolar pulses, the $\Delta \mathrm{V}_{\text {th }}$ are initially positive and eventually trend towards negative for all pulse widths.

For bipolar AC BTS, we find that instability also has a dependence on the operation frequency and that higher frequency causes more instability. This is an important issue which should be addressed for high-refresh rate displays. Upon changing the duty cycle of bipolar pulses from $50 \%$ to $10 \%$, we can suppress the $\Delta V_{\text {th }}$ for the same frequency. This shows that different pixel addressing methods, in the form of duty cycle control, may be viable for improving device stability.

\section{Acknowledgements}

We would like to thank Qualcomm Inc. for partial financial support of this study.

\section{References}

[1] K. Nomura, H. Ohta, A. Takagi, T. Kamiya, M. Hirano, and $\mathrm{H}$. Hosono, "Room-temperature fabrication of transparent flexible thin-film transistors using amorphous oxide semiconductors," Nature 432, No. 7016, 488-492 (2004).

[2] T. Kamiya, K. Nomura, and H. Hosono, "Present Status of amorphous In-Ga-Zn-O thin-film transistors," Sci. Technol. Adv. Mater. 11, 044305 (2010).

[3] C. J. Kim, D. S. Kang, I. Song, J. C. Park, H. Lim, S. Kim, E. Lee, R. Chung, J. C. Lee, and Y. Park, "Highly stable $\mathrm{Ga}_{2} \mathrm{O}_{3}-\mathrm{In}_{2} \mathrm{O}_{3}-\mathrm{ZnO}$ TFT for active-matrix organic light- emitting diode display application," in Proc. IEDM, 2006, pp. 1-4.

[4] T.-C. Fung, K. Abe, H. Kumomi, and J. Kanicki, "Electrical Instability of RF Sputter Amorphous In-Ga-ZnO Thin-Film Transistors," J. Display Technol. 5, 452 (2009).

[5] J. Y. Kwon, J. S. Sung, K. S. Son, T. S. Kim, M. K. Ryu, K. B. Park, Y. S. Park, S. Y. Lee, and J. M. Kim, "GaInZnO TFT for active matrix display," in $A M-F P D$ '08 Dig. Tech. Papers, 2008, p-. 287-290.

[6] Y. Ohto, Y. Chikama, T. Hara, Y. Mizuno, T. Aita, M. Takei, M. Suzuki, O. Nakagawa, Y. Harumoto, H. Nishiki, and N. Kimura, "Amorphous In-Ga-Zn-O TFT with high reliability," in $I D W$ '09 (The $16^{\text {th }}$ International Display Workshop), 1685-1688 (2009).

[7] A. Suresh and J. F. Muth, "Bias stress stability of indium gallium zinc oxide channel based transparent thin film transistors," Appl. Phys. Lett. 93, 093504 (2008).

[8] K. Nomura, T. Kamiya, and H. Hosono, "Interface and bulk effects for bias-light-illumination instability in amorphous-In-Ga-Zn-O thin-film transistors," J. Soc. Info. Display 18, 789 (2010).

[9] T.-C. Fung, K. Abe, H. Kumomi, and J. Kanicki, "DC/AC Electrical of R.F. Sputter Amorphous In-Ga-Zn-O TFTs," in SID Int. Symp. Dig. Tech. Papers 40, 1117 (2009).

[10] S.-J. Kim, S.-Y. Lee, Y. W. Lee, S.-H. Kuk, J.-Y. Kwon, and M.-K. Han, "Effect of Charge Trapping/Detrapping on Threshold Voltage Shift of IGZO TFTs under AC Bias Stress," Electrochem. Solid-State Lett. 15, H108-H110 (2012).

[11] S.-J. Kim, Y.-W. Lee, S.-Y. Lee, J.-S. Woo, J.- Y. Kwon, and M.-K. Han, "The Effect of AC Bias Frequency on Threshold Voltage Shift of the Amorphous Oxide TFTs," in SID Int. Symp. Dig. Tech. Papers 42, 1195 (2011).

[12] C. S. Chiang, J. Kanicki, and K. Takechi, "Electrical Instability of Hydrogenated Amorphous Silicon Thin-Film Transistors for Active-Matrix Liquid-Crystal Displays," Jpn. J. Appl. Phys. 37, 4704-4710 (1998). 